\title{
O papel do usuário na organização do setor de coleta de sangue do Hemonúcleo de Apucarana, Paraná, Brasil
}

\author{
The role of the user in the organization of the blood collection of the blood center of Apucarana, \\ Paraná, Brazil
}

Leonardo Di Colli

Liana L. Bassi

Claudete A. Omotto ${ }^{3}$

Lucia H. M. Rehme ${ }^{4}$

Tiemi Matsuo ${ }^{5}$
O Hemonúcleo de Apucarana faz parte da rede pública de bancos de sangue do estado do Paraná e tem como finalidade fornecer hemocomponentes com qualidade aos hospitais a ele conveniados. Com a proposta de atingir as metas do Ministério da Saúde em aumentar a quantidade de doadores espontâneos, o serviço tem se preocupado em buscar subsidios para melhora do processo de trabalho a fim de que este possa favorecer os objetivos de seu plano diretor. Para avaliar este processo, tornou-se necessário elaborar um instrumento de avaliação que possibilitasse identificar a percepção do doador em relação às diferentes etapas do processo de doação, bem como as áreas de insatisfação que se refletem em impedimentos para o retorno espontâneo deste doador. Este trabalho tem, portanto, o objetivo de demonstrar a satisfação dos doadores de sangue em relação às atividades executadas pelo Hemepar-Apucarana. Foram avaliadas questões como: orientações recebidas, horário de atendimento, ambiente fisico (limpeza, agradabilidade e cortesia) e equipe de trabalho. Todos os dados obtidos foram registrados num banco de dados e tabulados pela planilha Excel do Windows. Os dados determinados para avaliação foram classificados em ruim, regular, bom e ótimo, calculados em percentuais dispostos em tabelas elaboradas para sua visualização. Como resultado observou-se melhora da satisfação do doador em relação ao atendimento médico $(p=0,038)$ e diminuição da satisfação em relação aos equipamentos e vestuário dos funcionários $(p=0,012)$. Os demais itens não apresentaram diferenças estatisticamente significantes entre os dois períodos avaliados. Rev. Bras. Hematol. Hemoter. 2009;31(2):98-103.

Palavras-chave: Doador de sangue; processo de trabalho; avaliação; satisfação do doador.

\section{Introdução}

A descentralização dos serviços de hemoterapia foi legalmente amparada pela Portaria ${ }^{\circ} 07$ de 30 de abril de 1980, do Ministério da Saúde, que preconizava duas ações básicas: instituir o Sistema Nacional de Hematologia e Hemo- terapia e criar a Rede Nacional de Centros de Hematologia e Hemoterapia. ${ }^{1}$

O Centro de Hematologia e Hemoterapia do Paraná Hemepar - foi criado em 31 de março de 1982, como unidade prestadora de serviço da Secretaria de Estado da Saúde, com a finalidade de atender as necessidades de hemoterapia do

\footnotetext{
${ }^{1}$ Farmacêutico-Bioquímico e chefe do Hemonúcleo de Apucarana-PR.

${ }^{2}$ Assistente Social do Hemonúcleo de Apucarana e professora do Cesumar - Centro Universitário de Maringá-PR.

${ }^{3}$ Enfermeira do Hemonúcleo de Apucarana-PR.

${ }^{4}$ Assistente Social do Hemonúcleo de Apucarana-PR.

${ }^{5}$ Professora de Estatística do Curso de Mestrado da Universidade Estadual de Londrina-PR.
}

Hemonúcleo de Apucarana, Hemepar, Paraná

Correspondência: Leonardo Di Colli

Rua Antonio Ostrenski, 03 - Centro

86800-260 - Apucarana-PR - Brasil

E-mail:leodicolli@bwnet.com.br

Doi: 10.1590/S1516-84842009005000014 
estado do Paraná, em consonância com a Política Nacional de Sangue.

Atualmente a rede é composta de quatro hemocentros, tendo como coordenador o Hemocentro de Curitiba, sete hemonúcleos e 13 unidades de coleta e transfusão, sendo uma em cada município sede das Regionais de Saúde e duas unidades de âmbito municipal, compondo a rede Hemepar. Atende atualmente aproximadamente $60 \%$ dos leitos/SUS do estado, representando $50 \%$ das transfusões realizadas em 376 hospitais públicos, filantrópicos e privados conveniados ao SUS e onde se encontram implantadas 49 agências transfusionais. $^{2}$

O Hemonúcleo de Apucarana, fazendo parte do processo de descentralização da rede Hemepar, iniciou suas atividades em 1990 atendendo inicialmente os hospitais da sede da $16^{\mathrm{a}}$ Regional de Saúde de Apucarana, estendendo-se posteriormente para os demais 17 municípios que a ela pertencem, e tem a capacidade de coletar, fracionar, realizar testes sorológicos e distribuir sangue e seus componentes, sendo necessária uma média de 600 doadores/ mês para manutenção de seu estoque e garantir o fornecimento de sangue.

Enquanto instituição pública, segue as diretrizes da Política Nacional de Sangue e Hemoderivados, servindo de referência de qualidade nos procedimentos realizados. Dentre as metas preconizadas pelo Ministério da Saúde e do Plano Diretor da Rede Hemepar está a necessidade de aumentar a quantidade de doadores espontâneos, uma vez que no Brasil predomina a prática da doação de reposição, entendida como aquela doação solicitada aos "membros da família, ou a amigos de um paciente que precise de transfusão que doem sangue no intuito de suprir o estoque", como também atingir o índice de $60 \%$ de doadores de repetição, entendendo-se como repetição aquele doador que doou sangue no mínimo duas vezes nos últimos 13 meses. ${ }^{3}$ Está comprovado que a qualidade e segurança do sangue aumenta à medida que diminuem as doações de reposição ou com qualquer tipo de interesse em troca, aumentando a doação espontânea e de repetição. ${ }^{4,5}$ Tal fato se dá principalmente porque na doação de reposição, a principal motivação não está associada ao desejo de salvar vidas ou melhorar a qualidade de vida de outras pessoas e, por esta razão, podem omitir informações que os tornariam inaptos para a doação. De acordo com documento da Cruz Vermelha, ${ }^{6}$ embora os doadores de reposição de familiares e amigos sejam mais seguros do que os remunerados (prática exercida ainda em alguns países), os doadores de reposição para familiares apresentam maior incidência e prevalência de infecções transmissíveis pelo sangue, do que os voluntários, fato este que pode estar relacionado à pressão exercida para que doem.

Para se garantir uma doação contínua são necessárias ações que favoreçam a interação entre doador e instituição que consequentemente possa vir a gerar um processo de fidelização deste doador. Entretanto, a fidelização, entendida enquanto o retorno desse doador ao serviço habitual e espontaneamente, depende, entre outros fatores, da construção de relacionamento pessoal entre equipe de trabalho e doadores, além de um ambiente que inspire lealdade e confiança. ${ }^{7}$ Com esta premissa, o Hemonúcleo de Apucarana elaborou um instrumento de avaliação que possibilitou identificar a percepção do doador em relação às diferentes etapas do processo de doação, bem como as áreas de insatisfação que refletem impedimentos para o retorno deste doador espontaneamente.

A avaliação não é prática utilizada correntemente pelos serviços de saúde do país, constituindo ainda em uma área em desenvolvimento, tanto metodológico como conceitual, e faz parte das preocupações dos gestores do setor público e privado. ${ }^{8}$ Constitui uma etapa essencial do processo de planejamento e de administração no setor de saúde, pois levanta condições existentes, o processo de trabalho e os resultados, fazendo a comparação tanto com as condições e tecnologias previstas, quanto com os resultados e metas a serem alcançados para emissão de um juízo de valor e posterior tomada de decisão de maneira mais racional possível em face dos problemas enfrentados pelos serviços de saúde. ${ }^{9}$

Entendida dessa forma, a avaliação torna-se um processo dinâmico, integrado ao processo decisório, devendo estar presente nas diferentes esferas de gestão. Deve ser um processo permanente e sistemático, articulado às ações implementadas, com vistas a subsidiar definição de problemas, reorientar estratégias desenvolvidas, fornecer elementos para a transformação das práticas sanitárias, mensurar impactos das ações sobre a condição da vida de saúde das populações e sobre a organização dos serviços. Portanto, a avaliação torna-se etapa fundamental para gestão de serviços de saúde. ${ }^{10}$

Este trabalho tem, portanto, o objetivo de avaliar o nível de satisfação dos doadores de sangue em relação ao setor de coleta do Hemepar-Apucarana no ano de 2006 e 2007 para direcionamento da organização do serviço prestado, introduzindo procedimentos efetivos no processo de trabalho através de discussões subsidiadas pelos resultados, com vistas à fidelização dos doadores.

\section{Casuística e Método}

A pesquisa foi realizada em dois períodos, de janeiro a junho de 2006 e janeiro a setembro de 2007, através do instrumento de avaliação que consiste em um questionário semiestruturado composto por doze questões referentes às diferentes etapas da doação, desde a recepção, pré-consulta de enfermagem, triagem médica, coleta do sangue até o fornecimento do lanche ao doador. Também foram avaliadas questões como: orientações recebidas, horário de atendimento, tempo de espera, ambiente físico (limpeza, agradável) e equi- 
pe de trabalho, quantas doações já realizou e motivo da doação, além de contar com a presença de um espaço aberto para que o doador manifestasse sua opinião. Os questionários ficaram disponibilizados na sala de lanche, sendo esta a última etapa da doação, onde os doadores foram convidados a emitir sua opinião através do preenchimento do instrumento e depositá-lo em uma urna específica para este fim.

Todas as informações obtidas foram registradas num banco de dados e tabuladas pela planilha Excel do Windows. Os dados determinados para avaliação foram classificados em: ruim, regular, bom e ótimo e calculados em percentuais. Para visualização foram dispostos em tabelas. Os resultados foram comparados entre os períodos estudados, de janeiro a junho de 2006 e janeiro a setembro de 2007, utilizando-se o teste do qui-quadrado a nível de significância de 5\%.

\section{Resultados}

Conforme Tabela 1, cujo levantamento foi realizado entre janeiro a junho de 2006, verifica-se que, em relação ao atendimento da recepção, este foi considerado ótimo por $79 \%$ dos doadores que o avaliaram; o mesmo índice foi obtido quanto ao atendimento do auxiliar de enfermagem. $\mathrm{O}$ atendimento médico apresentou variações entre $6 \%$ como atendimento ruim, $10 \%$ regular, $23 \%$ bom e $61 \%$ ótimo. A sala de coleta foi avaliada como ótima por $83 \%$ dos doadores. No momento do lanche, o índice foi de $88 \%$ como de ótimo atendimento, sendo este o maior índice encontrado. O lanche foi avaliado como ótimo por $71 \%$ dos doadores. A Equipe apresentou um índice de $73 \%$ como de ótimo atendimento. Quanto ao tempo de espera, este apresentou uma distribuição nos resultados e um alto percentual de doado- res que não o avaliaram, contudo, aqueles que o consideraram ótimo, ou seja, permaneceram no local cerca de $30 \mathrm{mi}-$ nutos, corresponderam a $52 \%$, seguido de $7,5 \%$ no tempo de espera entre 45 minutos e 1 hora e 4\% superior a 1 hora. As informações foram consideradas ótimas por cerca de $71 \%$ dos doadores. $\mathrm{O}$ ambiente físico apresentou um índice de $85,5 \%$ que o consideraram ótimo. Quanto aos equipamentos e vestuário dos funcionários, estes foram avaliados como ótimos por $79 \%$ dos doadores.

Os resultados da avaliação compreendida entre os meses de janeiro a setembro de 2007 estão demonstrados na Tabela 2. Verifica-se que o atendimento ocorrido na recepção foi considerado ótimo por $73 \%$ dos doadores e para o atendimento de enfermagem este índice foi de $79 \%$. O atendimento médico foi classificado como ruim por $2,5 \%$ dos entrevistados, regular $7,5 \%$, bom $18 \%$, e ótimo por $72 \%$ deles. Um alto percentual de doadores considerou a sala de coleta como ótima, ou seja, $88 \%$, o mesmo acontecendo com o atendimento no momento do lanche, cujo índice foi de $89 \%$. Em relação ao lanche e a equipe cortês e agradável, estes valores variaram conforme observado na tabela. $\mathrm{O}$ tempo de espera cuja distribuição corresponde a $6 \%$ para os que permaneceram mais de 1 hora no aguardo o atendimento, $10 \%$ aguardaram 60 minutos, $11 \%$ aguardaram 45 minutos e $57 \%$, apenas 30 minutos. A clareza de informação foi considerada entre boa e ótima por $87 \%$ dos doadores. Cerca de $15 \%$ consideraram o horário de atendimento entre ruim e regular e $85 \%$ entre bom e ótimo. Em relação ao ambiente, $83 \%$ dos doadores o consideraram ótimo e quanto às vestimentas e equipamentos este índice foi de $71 \%$. Tendo como referência os tipos de doação, $60 \%$ dos doadores que responderam ao questionário eram voluntários

Tabela 1. Resultados da avaliação aplicada aos doadores de sangue do Hemonúcleo de Apucarana no período de janeiro a junho de $2006(n=349)$

\begin{tabular}{|c|c|c|c|c|c|}
\hline Questões & Ruim & Regular & Bom & Ótimo & Não respondeu \\
\hline 1. Atendimento na recepção & $2 \%$ & & $19 \%$ & $79 \%$ & \\
\hline 2. Atendimento do auxiliar de Enfermagem & & $2 \%$ & $19 \%$ & $79 \%$ & \\
\hline 3. Atendimento médico & $6 \%$ & $10 \%$ & $23 \%$ & $61 \%$ & \\
\hline 4. Atendimento na sala de coleta & & $2 \%$ & $15 \%$ & $83 \%$ & \\
\hline 5. Atendimento no momento do lanche & & $2 \%$ & $10 \%$ & $88 \%$ & \\
\hline 6. Lanche & & $2 \%$ & $25 \%$ & $71 \%$ & $2 \%$ \\
\hline 7. Equipe agradável e cortês & & $4 \%$ & $11,5 \%$ & $73 \%$ & $11,5 \%$ \\
\hline 8. Tempo de espera e permanência no serviço & $\begin{array}{c}+ \text { de } 1 \text { hora } \\
4 \%\end{array}$ & $\begin{array}{c}1 \text { hora } \\
7,5 \%\end{array}$ & $\begin{array}{c}45 \min \\
7,5 \%\end{array}$ & $\begin{array}{c}-30 \min \\
52 \%\end{array}$ & $29 \%$ \\
\hline 9. Clareza das informações sobre a doação & & $4 \%$ & $19 \%$ & $71 \%$ & $6 \%$ \\
\hline 10. Horário de atendimento & $6 \%$ & $7,5 \%$ & $17 \%$ & $63,5 \%$ & $6 \%$ \\
\hline 11. Ambiente físico do atendimento (limpo e agradável) & & & $9,5 \%$ & $86,5 \%$ & $4 \%$ \\
\hline 12. Equipamentos e vestuário dos funcionários & & $6 \%$ & $11,5 \%$ & $79 \%$ & $4 \%$ \\
\hline
\end{tabular}

Fonte: Registro de Dados do Hemonúcleo de Apucarana 
Tabela 2. Resultados da avaliação aplicada aos doadores de sangue do Hemonúcleo de Apucarana no período de janeiro a setembro de $2007(n=169)$

\begin{tabular}{|c|c|c|c|c|c|}
\hline Questões & Ruim & Regular & Bom & Ótimo & $\begin{array}{c}\text { Não } \\
\text { respondeu }\end{array}$ \\
\hline 1. Atendimento na recepção & $1 \%$ & $2 \%$ & $24 \%$ & $73 \%$ & \\
\hline 2. Atendimento do auxiliar de Enfermagem & $0,5 \%$ & $0,5 \%$ & $20 \%$ & $79 \%$ & \\
\hline 3. Atendimento médico & $2,5 \%$ & $7,5 \%$ & $18 \%$ & $72 \%$ & \\
\hline 4. Atendimento na sala de coleta & & $1 \%$ & $11 \%$ & $88 \%$ & \\
\hline 5. Atendimento no momento do lanche & & $1 \%$ & $10 \%$ & $89 \%$ & \\
\hline 6. Lanche & & $2 \%$ & $20 \%$ & $76 \%$ & $2 \%$ \\
\hline 7. Equipe agradável e cortês & & $2 \%$ & $15 \%$ & $77 \%$ & $6 \%$ \\
\hline 8. Tempo de espera e permanência no serviço & $\begin{array}{c}\text { + de } 1 \text { hora } \\
6 \%\end{array}$ & $\begin{array}{c}1 \text { hora } \\
10 \%\end{array}$ & $\begin{array}{c}45 \min \\
11 \%\end{array}$ & $\begin{array}{c}-30 \min \\
57 \%\end{array}$ & $16 \%$ \\
\hline 9. Clareza das informações sobre a doação & $1 \%$ & $10 \%$ & $11 \%$ & $57 \%$ & $16 \%$ \\
\hline 10. Horário de atendimento & $4 \%$ & $11 \%$ & $17 \%$ & $62 \%$ & $6 \%$ \\
\hline 11. Ambiente físico do atendimento (limpo e agradável) & & $1 \%$ & $11 \%$ & $83 \%$ & $5 \%$ \\
\hline 12. Equipamentos e vestuário dos funcionários & $2,5 \%$ & $2,5 \%$ & $21,5 \%$ & $71,5 \%$ & $2 \%$ \\
\hline
\end{tabular}

Fonte: Registro de Dados do Hemonúcleo de Apucarana

Tabela 3. Comparação dos resultados da avaliação aplicada aos doadores de sangue do Hemonúcleo de Apucarana, entre os períodos de janeiro a junho de 2006 e janeiro a setembro de 2007

\begin{tabular}{lccc}
\hline \multicolumn{1}{c}{ Questões } & $\mathrm{x}^{2}$ & $\mathrm{GL}$ & Valor de $\mathrm{p}$ \\
\hline 1. Atendimento na recepção & 2,77 & 2 & 0,250 \\
2. Atendimento do auxiliar de Enfermagem & 0,53 & 2 & 0,767 \\
3. Atendimento médico* & 6,52 & 2 & 0,038 \\
4. Atendimento na sala de coleta & 2,14 & 2 & 0,343 \\
5. Atendimento no momento do lanche & 1,95 & 2 & 0,377 \\
6. Lanche & 1,95 & 2 & 0,377 \\
7. Equipe agradável e cortês & 1,71 & 2 & 0,426 \\
8. Tempo de espera e permanência no serviço & 1,47 & 2 & 0,478 \\
9. Clareza das informações sobre a doação & 3,62 & 2 & 0,163 \\
10. Horário de atendimento & 0,15 & 2 & 0,919 \\
11. Ambiente físico do atendimento (limpo e agradável) & 4,74 & 2 & 0,094 \\
12. Equipamentos e vestuário dos funcionários ${ }^{* \star}$ & 8,80 & 2 & 0,012 \\
\hline
\end{tabular}

* Houve diminuição de Ruim+Regular e aumento de Ótimo $(p=0,038)$

** Diminuiu Ótimo e aumentou o Bom $(p=0,012)$

ou espontâneos, seguido de $15 \%$ para reposição e 1,7\% por convocação do Banco de Sangue (prática comum quando se necessita de um tipo específico de sangue). A metade dos doadores já havia doado mais de 4 vezes. Neste item, $25 \%$ dos doadores estavam comparecendo no serviço pela primeira vez.

$\mathrm{Na}$ Tabela 3, considerando-se a junção das categorias Ruim e Regular, demonstra que nos ítens 3- Atendimento Médico e 12- Equipamentos e Vestuário houve diferença significativa quando comparado os dois períodos.

\section{Discussão}

Para o Atendimento Médico verificou-se que houve melhora na avaliação, pois a categoria Ruim diminuiu de $6 \%$ em 2006 para 2,5\% em 2007, a categoria Regular também diminuiu de $10 \%$ para $7,5 \%$, enquanto a Ótimo aumentou de $61 \%$ em 2006 para $72 \%$ em 2007 , sendo estas diferenças consideradas estatisticamente significantes $(\mathrm{p}<0,038)$.

Em relação aos equipamentos e vestuário dos funcionários houve uma diminuição da satisfação dos usuários nesta avaliação, pois ocorreu que a frequência da categoria Ótimo diminuiu de $79 \%$ para $71 \%$, e aumentou a categoria Bom, de $11,5 \%$ para $21,5 \%$, diferenças estas consideradas estatisticamente significantes $(\mathrm{p}<0,012)$.

Para os demais ítens não houve diferença significativa.

A implantação de um processo de avaliação do grau de satisfação do usuário do serviço de Hemoterapia de Apucarana trouxe avanços importantes no aperfeiçoamento da gestão. A satisfação do doador de sangue está positivamente relacionada à intenção de retorno para uma futura doação. ${ }^{11}$

Dentro de um processo contiínuo na busca pela qualidade, as avaliações não têm como objetivo verificar com generalidade o nível de satisfação do doador, mas conhecer especificamente aqueles ítens que merecem atenção. ${ }^{12} \mathrm{Neste}$ 
sentido, esta avaliação apontou insatisfações conforme demonstrado.

Quando analisadas as Tabelas 1 e 2, verifica-se que houve ótima avaliação quanto ao atendimento na recepção, atendimento sala de coleta, pré-consulta, qualidade e momento do lanche, tratamento dispensado pela equipe, com cordialidade e cortesia no atendimento e clareza e precisão nas informações prestadas, nos dois períodos em estudo. $\mathrm{Na}$ demonstração destes ítens na Tabela 3, verifica-se que não houve diferença estatisticamente significante, o que vem demonstrar que nestas áreas poucos são os problemas a serem resolvidos.

Em relação ao atendimento médico, a insatisfação observada em 2006 implicou a adoção de algumas ações específicas para este setor, como discussão do acolhimento proporcionado ao doador na triagem clínica, agendamento prévio de grupo de doadores após horário comercial e coleta de sangue uma vez por mês aos sábados em parceria com Secretarias Municipais de Saúde na área de abrangência da $16^{\mathrm{a}}$ Regional de Saúde. Essas medidas se refletiram na alteração dos índices no ano de 2007, observando-se uma melhora significativa neste indicador, cuja comparação entre os dois períodos foi considerada estatisticamente significativa.

Quanto às avaliações referentes ao tempo de espera e horário de atendimento, também houve um alto grau de insatisfação, uma vez que é ruim para cerca de $6 \%$ e regular para $7,5 \%$, o que impede o retorno dos doadores, porém esta diferença não foi considerada significativa $(\mathrm{p}=0,478)$. Dados semelhantes em relação ao tempo para doação foram observados no Centro Regional de Hemoterapia de Ribeirão Preto. ${ }^{13}$ Tal fato pode ser justificado, em parte, pela sobrecarga do profissional médico no horário da triagem médica, dado o grande número de doadores que, em sua maioria, comparecem em grupos, ocasionando a espera.

$\mathrm{Na}$ avaliação do equipamento e vestuário dos funcionários, houve diminuição da satisfação do usuário entre os dois períodos estudados, o que sugere ter havido nesse interregno desgaste natural dos equipamentos, além de uma acomodação por parte da equipe em relação a sua apresentação diante dos doadores. Neste sentido, novas mudanças tiveram de ocorrer para que a qualidade observada na primeira avaliação fosse suplantada.

No espaço aberto do questionário, os doadores apresentaram críticas, sugestões e elogios à equipe; tal questionário foi utilizado pelos usuários principalmente para apontar o bom atendimento realizado na instituição, reforçando as conclusões da pesquisa.

Além de revelar nós críticos no atendimento, o trabalho demonstrou que o processo de doação do Hemonúcleo de Apucarana possui eficácia, considerando-se que a qualidade se apresentou entre boa e ótima, motivando assim a equipe a manter o padrão de atendimento. Ademais, contribuiu para despertar para a consciência da responsabilidade de todos os membros, da recepção até o lanche, na efetivação de maior segurança na coleta de sangue, bem como de seus doadores.

\section{Considerações finais}

Os resultados obtidos deste estudo proporcionaram à instituição avaliar as estratégias utilizadas para implementação e ou superação dos pontos críticos bem como sua solução. As principais ações consistiram de reuniões de funcionários, oficinas de integração da equipe, oficina de educação permanente em saúde, encontros técnicos regionais e encontros de doadores. Revelou a necessidade de introduzir o processo de avaliação interna de forma permanente com o levantamento de outros indicadores de qualidade com vistas à excelência, incluindo atividades de monitoramento que permitam intervir no processo de trabalho, corrigindo os rumos cada vez que desvios são detectados, considerando a relevância do atendimento prestado na instituição para a vida das pessoas.

O usuário demonstrou ser uma importante fonte de informações e a principal fonte para avaliar o atendimento a ele prestado. Esta contribuição dá aos gestores de saúde caminhos para obtenção da melhor qualidade dos serviços e consequentemente maior satisfação aos usuários.

\section{Abstract}

The hemocenter of the city of Apucarana, PR, Brazil, is part of Paraná State's Network of Blood Banks. Its purpose is to provide member hospitals with high-quality hemo-components. Proposing to fulfill the Brazilian Health Ministry's goals to increase the amount of first-time donors, Hemepar-Apucarana has been concerned with acquiring subsidies to improve its procedures so that it can benefit from the application of its own main directive. To evaluate this process, it is necessary to elaborate an instrument that can assist with identifying the donor's perception in regards to the different stages associated with blood donation, especially areas of dissatisfaction, and reflecting also on the areas that hinder the return of first-time donors. This work has thus the purpose of demonstrating blood donors' degree of satisfaction toward the activities of Hemepar - Apucarana. Issues such as the following have been evaluated: Instructions, reception schedule, physical ambiance (cleanness, coziness), and staff. All data has been saved in a Microsoft Windows Excel chart. The points under evaluation have been rated by actual donors from a range of bad, average, good and excellent pertaining to service and procedure. Percentages have also been calculated and plotted in graphs for visual comparison. As a result it was observed an improvement of the donor related to the medical service $(p=0,038)$ and decreasing of satisfaction related to the equipments and employees' vestry $(p=0,012)$. The other items didn't present significant differences between both periods. Rev. Bras. Hematol. Hemoter. 2009;31(2):98-103.

Key words:Blood donor; working process; evaluation; blood donor satisfaction. 


\section{Referências Bibliográficas}

1. Brasil. Ministério da Saúde. Portaria Interministerial $n^{\circ} 7$ de 30 de abril de 1980. Aprova as diretrizes básicas do Programa Nacional de Sangue e Hemoderivados (Pró-Sangue). Brasília; 1980.

2. Paraná. Secretaria de Estado da Saúde. Centro de Hematologia e Hemoterapia do Paraná (Hemepar). Relatório de Gestão. Curitiba; 2006

3. Paraná. Secretaria de Estado da Saúde. Plano Diretor de Sangue do Estado do Paraná. Curitiba; 2006.

4. Organização Pan-Americana da Saúde (OPAS). Federação Internacional das Sociedades da Cruz Vermelha e do Crescente Verme1ho. Fazendo a diferença: captando doadores de sangue voluntários, não remunerados. Brasília; 2004.

5. Organização Mundial da Saúde (OMS). Segurança do sangue: artigo para apoio de Programas Nacionais de Sangue; 1999. [citado 2008 set 24]. Disponível em: http://www.who.int/bloodsafety/ transfusion_services/en/Blood Safety_Port.pdf.

6. Federação Internacional de Sociedades de la Cruz Roja y de la Media Luna Roja. Reclutamiento de donantes de sangre. Boletín Internacional IRFC 2008; 101(83): 1-19. [citado 2008 set 24]. Disponível em: http://www.ifrc.org/Docs/pubs/health/blood/dri101-sp.pdf.

7. Ludwig ST, Rodrigues ACM. Doação de sangue: uma visão de marketing. Cad Saúde Pública. 2005; 2(3): 932-9.

8. Di Colli L. A gestão plena do Sistema Municipal: uma avaliação em Apucarana - PR [Dissertação]. Londrina: Universidade Estadual de Londrina. 2006. [citado 2008 jul 14]. Disponível em: http://ccs.uel.br/pos/mestrado/mural/dissertacao/Leonardo/Leonardo completo.pdf.

9. César CLG, Tanaka OY. Inquérito domiciliar como instrumento de avaliação de serviços de saúde: um estudo de caso na região sudeste da área metropolitana de São Paulo, 1989-1990. Cad Saúde Pública. 1996; 12(2): S59-S70.

10. Solla JJS, Santos FP. Avaliação da implantação das ações de controle, avaliação e auditoria na Gestão Plena do Sistema Municipal de Saúde. In: Brasil. Ministério da Saúde. Experiências inovadoras no SUS: relato de experiências/gestão dos serviços de saúde. Brasília; 2002; p. 361-91.

11. Nguyen DD, Devita DA, Hirschler NV, Murphy EL. Blood donor satisfaction and intention of future donation. Transfusion. 2008;48(4):742-8

12. Borges VL, Martinez EZ, Bendini MH, Costa MAGF, Ferreira SCL. Avaliação da fidedignidade de um instrumento voltado à satisfação do doador de sangue. Rev Bras de Epidemiologia. 2005; 8(2):177-86

13. Borges VL, Valente UB, Bendini MH, Jacob APP, Bertoso SPS, Zanelli APRD, et al. Avaliação da satisfação do doador de sangue Rev Bras Hematol Hemot. 2007;29(3):272.

Avaliação: Editor e dois revisores externos

Conflito de interesse: não declarado

Recebido: 13/04/2008

Aceito após modificações: 20/12/2008 